\title{
Teaching Content and Method of Mechanical Optimization Design in Independent College
}

\author{
Youxin Luo \\ College of Mechanical Engineering \\ Hunan University of Arts and Science \\ Changde, 415000,P.R.China \\ e-mail: LLYX123@126.com
}

\author{
Zheming HE \\ College of Mechanical Engineering \\ Hunan University of Arts and Science \\ Changde, 415000, P.R.China \\ e-mail: hzming@126.com
}

\begin{abstract}
Considering the characteristics of the students of the Independent College, the problems existed in teaching Mechanical Optimization Design, main and important contents of the course is emphasized, teaching methods is improved, and the test and exam ways is reformed. The teaching practice showed that students had a deeper understanding of the optimal design, and mastered the basic principles of the optimal methods, and teaching effectiveness was enhanced, the quality of education was improved.
\end{abstract}

Keywords-Course;Teaching method;MechanicalOptimization Design

\section{INTRODUCTION}

Mechanical optimization design is a selected course for students who major in mechanical engineering. The purpose of this course is to enable students to establish the optimal design thinking, to grasp the basic concepts and methods, and the initial capacity to solve the problem of mechanical optimization. Through learning the course, students can not only master the knowledge of higher mathematics, mechanical design and programming language and, more important, can gradually master the optimization of the design ideas and methods in practice, to develop students' ability to solve practical problems. Therefore, this practical course aims to develop student's innovative spirit and the practical ability. This paper discusses the teaching and learning for the last few years, and offers a few ideas about the course.

\section{CHARACTERISTICS OF THE STUDENT IN INDEPENDENT \\ COLLEGE}

Less than ordinary undergraduate college students in learning, the students in independent college are poor in selfcontrol and self-monitoring. Because of better family conditions in the previous life and long-term care by the elders, the students are weak at self-care ability. Teachers in teaching should give full play to their own professional advantage to focus on student learning guidance, supervision. Firstly, it should allow the students to know about the specialty, master the scientific learning and research methods. Secondly, it should be answering questions for students to solve practical difficulties encountered by students in learning. Thirdly, urge students complete learning tasks efficiently, to broad their knowledge. At the same time, the personality is more prominent, it should be noted that how to highlight the personality of the students.

\section{PROBLEMS EXISTED IN TEACHING}

From the situation of the past few years, it is found that many students feel that the course content is boring, and not interested in learning mechanical optimization design courses. There are two major problems: First, in the classroom teaching, mathematical knowledge learned have been forgotten mostly, and even some students say "I do not know where to begin the higher mathematics and linear algebra". Second, in the practice session, it is very hard for them to use the programming language learned to write optimized design program, almost all of the students involved in the mechanical optimization do not know the design calculation methods.

\section{MAIN AND IMPORTANT CONTENTS OF THE COURSE}

Mechanical Optimization Design includes the following three aspects: the establishment of a mathematical model. Select the appropriate optimization methods and select the appropriate program. Mechanical optimization design is the application of mathematical methods to seek the optimal solution of the mechanical design. So, we must first establish a corresponding mathematical model based on the actual problem, which uses mathematical form to describe the actual engineering design problems. We need to use appropriate knowledge to determine the boundary conditions and the goal to determine the relationship between the variables. Once the mathematical model established, the problem of mechanical optimization becomes a mathematical problem. The optimization problem can be solved by programming technique, by selecting or writing a computer program, to determine the optimal design parameters.

In recent years, mechanical optimization design has been a selected course for students in many independent colleges. The textbooks have been published in various versions and different arrangement of contents. As a selected course for independent college, the main contents are: (1) The basic concepts, common terms. Mainly include the optimization problem instance, the mathematical model and the basic solution. (2) The basic knowledge of mathematics, including: derivatives in higher dimensions, directional derivative and 
gradient; the Taylor's expansion; convex; convex functions; the extreme condition of optimization problems. (3) The one -dimensional method: golden section method; (4) Unconstrained optimization problems. (5) The constrained stochastic optimization methods, including random direction method, compound form method, penalty function method, and so on.(6) The discrete variable optimum design; (7) The application example of optimum design, software programming and site commissioning.

For these students, the learning should not focus on theoretical, but application. The application to optimize the design is of the essence, optimization design method for the selection and application of techniques in the teaching process, and always adheres to the teaching focus.

Therefore, the importance of each part in the teaching process should be focused. Focus on teaching parts (1) and (2) of the contents, its purpose is to enable students to master the basic concepts and mathematical knowledge, lay the foundation for the later chapters. Although many mathematical problems involved in this part, we should start with the geometric interpretation of the mathematical concepts and to illustrate some of the basic concepts of optimization methods, but not focus on the mathematical formula. For the extreme conditions of optimization problems, it's better to depict the several situations may be encountered by graphic, that may be encountered in several situations, to illustrate the local minimum point and global minimum point. Kuhn-Tucker conditions can be ignored, because it can not use to be applied to determine whether a certain point is a global minimum point in engineering practice. The relationship between the design variables, objective function and constraints, and the optimal solution are clearly expressed in the plane coordinate system out by graphical method, which helps students enhance perceptual knowledge, deepen the understanding of the design process. Highlighting the contents of the identification of search intervals and golden section method when teaching the onedimensional method, then, students can write a computer program via a flow chart. Then introduce the basic principle of the spline interpolation simply. In the content of the unconstrained optimization method, we choose the steepest descent algorithm, coordinate reiterative method and Newton's method for highlights, which are the basis of other optimization methods, and the basic idea and calculation process are relatively simple. It is very useful for students to further understand other optimization methods after grasping this content. Other methods are use as references for choosing optimization methods only, describing the principles and characteristics simply. In the content of the constrained stochastic optimization methods, we highlight the random direction method, compound form method, penalty function method, and focus on the basic principle, procedure and flow chart, so that students have a basic understanding of constrained optimization method for solving problems. For discrete variable optimum design, we only clarify the concept and implement in software. At the last, we choose one or two mechanical optimization design examples to explain the establishment of the mathematical model as well as the application of software to achieve the whole calculation process then modifies some parameters as exercises.

\section{IMPROVEMENT OF TEACHING METHODS}

\section{A. Theory with practice, improve teaching methods}

Traditionally the theoretical analysis, formula deduction and practical calculation procedures are often presented in class, but rarely applied in an actual project. So what the students feel in the mechanical optimization design class is abstract theory, not pleasure about learning engineering examples. Students in class even after the examination still do not know where the learned knowledge can be applied to, and the low learning atmosphere lead to fruitless and frustrating. In fact, every design method has its corresponding practical application. As long as teachers in the classroom describe the background and practical applications, the students' learning desire will be stimulated, and the design method will be more attracted. Then, students will have a deeper understanding of the optimal design methods. In a word, from an actual engineering problem, describe the background and practical applications, this new teaching method will lead to an effective class.

\section{B. Build an actual model}

After studying Mechanics and Optimization Design, a model building competition can be organized. Take use of paper and double-sided tape as building material, students can build a bridge, a tall building, even a tunnel. This kind competition will be conducive to develop students' ability of innovation and practice, and problem consciousness. First of all, the students were asked to query structure information and pictures of various structures, go through all related reference material and papers wrote by their teachers, know about the format of scientific papers. Then teachers were asked to guide students to understand the basic concept of the structural design, methods and processes, introduce some basic skills and methods of the design of the paper structure, and apply the optimal design theory to actual design. A combination of research, teaching and production, let the gifted students take part in the teachers' research, establish the optimization model from the real products, cultivate innovative talents which really meet the needs of industry and practice. After completing the model, from the appearance, structure and load-bearing test, and whether the use of optimization theory is correct and reasonable to assess and score the model respectively, its results can also be used as part of the final score of this course. Through such activities, you can make the students interested in the optimization design, not only enlighten the students' innovative thinking to develop practical ability, and will promote unity between the students. The structural design involves a very wide range of knowledge, so that such activities also enable students to understanding of expertise deeper in all aspects. There, the activities stir up more interest of the students in technological innovation, promote a combination of textbook knowledge and practice. 


\section{Programming techniques}

For the mechanical optimization design courses, the emphasis on teaching the theory is impossible to get a good teaching effect, there must be some hours on the computer exercises, the general practice is to arrange one-third of the class time on the computer exercises. Languages like MATLAB, LINGO are usually used on programming exercises, and the exercise problems are among all chapters.

Before learning this course, the students already have some programming experiences; teachers would better to provide students with some lessons and skills on programming, some reference and classic source files in class. Contrast MATLAB, LINGO programming to reduce the difficulty. A source file can be provided to read, and learn how to use this source to obtain an optimal solution. And then they were asked to write program by themselves. Before the computer exercises, teacher should explain the experimental methods and flow diagram. In the computer experimental class, while guiding students to complete the experimental subject, the instructor let students who finish the experiment and not yet completed discuss each other, to find out problems, explore the correct method to improve their program by themselves.

\section{ASSESSMENT OF LEARNING OUTCOMES}

In this course, students' final score is divided into two parts: the usual performance and the last paper. And the usual performance accounts to $40 \%$, consists of four parts: attendance, classroom performance score, assignment score and experimental results, which focuses on the learning steps, rather than results.

A student who ask and answer questions positively in class will get a high score in classroom performance. Experimental results were comprehensive assessment by the content of the programming and experimental reports, so that good experimental attitude will be encouraged. The usual performance does not meet requirement can't permit to pass this course. It is different from the conventional mode, which is to contribute to cultivate the innovation consciousness and ability.

When we exam the current educational system, the situation is that students still have to withstand the pressure of the examination. This kind of traditional examination and assessment just focuses on the assessment of student knowledge levels, rather than focus on the assessment of innovative ability and practical ability. So, we test students in the end of the course by writing papers and building models, which played a positive role in developing innovative consciousness and ability, and students loved it. Considering they can solve some actual problem after learned the Mechanical Optimization Design, above assessment methods were implemented

\section{CONCLUSIONS}

Considering the characteristics of the student in Independent College and problems existed in teaching Mechanical Optimization Design, we redefine main and important contents of the course, improve teaching methods, reform the test and exam ways. The teaching practice shows that students had a deeper understanding of the optimal design, and mastered the basic principles of the optimal methods, and teaching effectiveness was enhanced, the quality of education was improved.

\section{ACKNOWLEDGMENT}

This research is supported by the grant of the 12th FiveYear Plan for the construct program of the key discipline (Mechanical Design and Theory) in Hunan province (XJF2011[76]).

\section{REFERENCES}

[1] J.M. Sun, Mechanical Optimization Design,Beijing:China Machine Press, 2008,pp.1-50.

[2] D.Y.Xue, MATLAB in Higher Applied Mathematics, Beijing:Tsinghua University press, 2008,pp.1-70.

[3] J.X. Xie, Y. Xue, Optimization Modeling with LINDO/LINGO, Tsinghua University press, 2005,pp.20-60. 